\title{
On Optimized Memoryless Relaying Functions for the Two-Way Relay Channel
}

\author{
Michael Heindlmaier, Onurcan İşcan, Ronald Böhnke and Christoph Hausl \\ Institute for Communications Engineering, Technische Universität München, Munich, Germany \\ Email: \{michael.heindlmaier, onurcan.iscan, ronald.boehnke, christoph.hausl\}@tum.de
}

\begin{abstract}
The separated restricted two-way relay channel (TWRC) has two sources that are outside of radio range and must communicate with the help of a relay. The relay is limited to perform memoryless operations on its received symbols to compute its channel input. Relay functions optimizing the achievable sum rate for both sources are investigated for binary antipodal signaling and pulse amplitude modulation (PAM). The results are compared to the performance of Amplify \& Forward (AF), Estimate \& Forward (EF) and Detect \& Forward (DF).
\end{abstract}

\section{INTRODUCTION}

The two-way relay channel (TWRC) incorporates challenging problems such as multiple access, broadcast and coding with side information. It is a suitable model for many applications, such as satellite communication. In this work, we focus on the separated restricted TWRC where the source nodes cannot hear each other's transmissions and input symbols are chosen independently.

Rankov et al. [1] evaluated achievable rate regions for the TWRC by using classical relaying strategies such as Decode \& Forward, Compress \& Forward and Amplify \& Forward. Apart from these schemes, novel relay strategies based on lattice coding [2]-[4] and nonlinear memoryless relaying [5]-[9] have been introduced.

For practical systems, Amplify \& Forward is a popular choice because it is independent of the modulation and coding scheme of the sender and does not require decoding operations at the relay. Motivated by the reduced complexity and delay involved, we concentrate on memoryless relaying.

\section{A. Memoryless Relaying}

Memoryless [5] or instantaneous relaying [6], [10] refers to the case where received symbols are forwarded on a symbol-by-symbol basis. The relay transmits an analog symbol depending on a single received symbol.

For example, Amplify \& Forward (AF) [11] has the relay linearly scale the received symbol. In general, nonlinear relay functions improve performance [5]: For example, for BPSK signaling, the binary XOR-operation turn out to be a good choice. This concept, often called physical layer network coding [7] or analog network coding [8] can be generalized to higher order modulation schemes.

For the one-way Gaussian relay channel without direct link, the optimal memoryless relay operation in terms of minimal uncoded bit error rate for binary antipodal signaling was derived in [12]. The authors of [5] extended these results to the separated TWRC and compared different approaches in terms of bit error rate. Minimum mean square error (MMSE) forwarding for the one-way relay channel was considered both without [13] and with [14] a direct link between source and destination. The technique is also referred to as Estimate \& Forward $(\mathrm{EF})$. If the relay performs hard decisions on the received symbol, the corresponding memoryless technique is called Detect \& Forward (DF).

In both [13] and [5] EF almost maximizes the mutual information for the one-way relay channel and uncoded bit error probability for the TWRC, respectively. Our results suggest that EF also almost maximizes the mutual information for the TWRC in some cases. In [15], the authors investigated the performance of $\mathrm{EF}$ in a coded system and considered further implementation aspects.

\section{B. Contribution}

Our goal is to find relay functions that maximize mutual information expressions for both node pairs. We numerically optimize relaying functions for different scenarios and modulation formats, extending ideas from [6] to discrete pulse amplitude modulation (PAM). Memoryless relaying techniques such as $\mathrm{AF}, \mathrm{EF}$ and $\mathrm{DF}$ are compared in terms of their achievable sum rate.

\section{SYSTEM MODEL}

Consider two source nodes $\mathrm{T}_{1}$ and $\mathrm{T}_{2}$ that communicate with each other via a relay but cannot hear each other's transmissions. This channel model is sometimes called a separated TWRC [16]. The messages $W_{1} \in\left\{1,2, \ldots, 2^{n R_{1}}\right\}$ and $W_{2} \in\left\{1,2, \ldots, 2^{n R_{2}}\right\}$ shall be exchanged in $n$ channel uses. These messages are encoded in codewords $X_{1}^{n} \in \mathcal{X}_{1}^{n}$ and $X_{2}^{n} \in \mathcal{X}_{2}^{n}$, which are functions of $W_{1}, W_{2}$, respectively. All devices operate in full-duplex mode. Note that the system model can easily be extended to the half-duplex case as well. The received signal after the matched filter at the relay is

$$
Y_{r, t}=X_{1, t}+X_{2, t}+Z_{r, t}=V_{t}+Z_{r, t}
$$

for time instant $t=\{1,2, \ldots, n\}$, where $X_{j, t} \in \mathcal{X}_{j}$ is the channel input for source $\mathrm{T}_{j}$ and $Y_{r, t} \in \mathcal{Y}_{r}$ the output at the relay. $V_{t}$ denotes the sum signal $X_{1, t}+X_{2, t}$, its set $\mathcal{V}$ is determined by $\mathcal{X}_{1}$ and $\mathcal{X}_{2}$. We consider one-dimensional modulation schemes. $Z_{r, t} \sim \mathcal{N}\left(0, N_{r}\right)$ denotes a real-valued Gaussian noise random variable. The relay processes only a 
single receive symbol to a single transmit symbol via the memoryless relay function $f$ :

$$
f: \mathcal{Y}_{r} \rightarrow \mathcal{X}_{r}
$$

We refer to $f$ as the relay mapping. The output of the mapping is then broadcasted to both sources. For simplicity, we assume in Eq. (2) that the relay mapping operates noncausally; this is only to simplify notation.

The received signal at source $\mathrm{T}_{j}$ is

$$
Y_{j, t}=f\left(Y_{r, t}\right)+Z_{j, t}
$$

where $Z_{j, t} \sim \mathcal{N}\left(0, N_{j}\right)$. The average transmit power of the sources and the relay is given by $P_{j}=\mathbb{E}\left\{X_{j, t}^{2}\right\}$, $P_{r}=\mathbb{E}\left\{f^{2}\left(Y_{r, t}\right)\right\}$, respectively. In the following, we omit the time index $t$, as we always consider one symbol at a specific time instant.

The channels in Eqs. (1) and (3) are additive white Gaussian noise (AWGN) channels with channel gains equal to one. We do not consider different channel gains in our model, as they can be expressed as different power and noise levels of the nodes.

\section{OPTIMIZED SCHEMES}

\section{A. Continuous Problem}

The authors in [6] optimize the rate region achieved with memoryless relaying for the two-way channel and Gaussian signaling. In this work, we focus on optimized mappings for pulse-amplitude modulation (PAM). We take BPSK and 4PAM as concrete examples.

Shannon showed in [17] that the rate region $\mathcal{R}$ is achievable for the memoryless two-way channel, where $\mathcal{R}$ denotes the set of all rate tupels $\left(R_{1}, R_{2}\right)$ satisfying

$$
\begin{aligned}
& 0 \leq R_{1}<I\left(X_{1} ; Y_{2} \mid X_{2}\right) \\
& 0 \leq R_{2}<I\left(X_{2} ; Y_{1} \mid X_{1}\right)
\end{aligned}
$$

for the product distribution $p\left(x_{1}, x_{2}\right)=p\left(x_{1}\right) p\left(x_{2}\right)$. If the sum rate is chosen as the measure of performance, a utility can be defined as

$$
U(f)=I\left(X_{1} ; Y_{2} \mid X_{2}\right)+I\left(X_{2} ; Y_{1} \mid X_{1}\right) .
$$

The relay mapping effectively changes the channel $p\left(y_{1}, y_{2} \mid x_{1}, x_{2}\right)$, so the optimization problem as in [6] can be stated as

$$
\begin{array}{cl}
\text { maximize } & U(f) \\
\text { subject to: } & \mathbb{E}\left\{f^{2}\left(Y_{r}\right)\right\} \leq P_{r} .
\end{array}
$$

The last inequality restricts $f: \mathbb{R} \rightarrow \mathbb{R}$ to satisfy the relay's power constraint.

The problem in Eq. (6) is a variational problem that seems to be analytically intractable. Also, determining $U(f)$ analytically for a given relay mapping $f$ can be a difficult problem: A closed form solution for the integral that determines the conditional distributions $p\left(y_{2} \mid x_{2}\right)$ and $p\left(y_{1} \mid x_{1}\right)$ is available only in special cases. We thus focus on numerically solving a discretized approximation of problem (6).

\section{B. Discretized Approximation}

A digital implementation of the memoryless relay function has received symbols at the relay analog-to-digital (AD) converted and passed into a digital signal processor. The corresponding output symbol is then digital-to-analog (DA) converted and passed to the amplifier. The cardinalities of all random variables are finite and represent the resolution of the $\mathrm{AD}$ and DA converters, respectively.

In order to have a finite-dimensional optimization problem over a continuous space, we require the range of $f$ to be the real numbers, so $f: \mathcal{Y}_{r} \rightarrow \mathbb{R}$. The approach presented can thus be seen as the limit of a finite-precision AD converter and infinite precision DA converter.

The relay mapping $f$ can thus be represented by a vector $\mathbf{f}=\left(f_{i}\right)_{i=1, \ldots,\left|\mathcal{Y}_{r}\right|} \in \mathbb{R}^{\left|\mathcal{Y}_{r}\right|}$. Each possible quantized receive value $y_{r i} \in \mathcal{Y}_{r}$ is mapped to $f_{i}$, i.e. $f_{i}=f\left(y_{r i}\right)$.

Due to the quantization at the receiver, the conditional probability densities convert to probability mass functions. Assuming a uniform quantization with interval length $\Delta$, we can express the conditional probability mass function for the AWGN channel as

$p\left(y_{r} \mid x_{1}, x_{2}\right)=Q\left(\frac{y_{r}-\Delta / 2-\left(x_{1}+x_{2}\right)}{\sqrt{N_{r}}}\right)-Q\left(\frac{y_{r}+\Delta / 2-\left(x_{1}+x_{2}\right)}{\sqrt{N_{r}}}\right)$ for each $y_{r} \in \mathcal{Y}_{r}$, where $Q(x)=\frac{1}{\sqrt{2 \pi}} \int_{x}^{\infty} \exp \left(-\frac{z^{2}}{2}\right) \mathrm{d} z$.

At the boundaries we obtain slightly different expressions taking into account the overload terms. Similarly, we have $p\left(y_{j} \mid y_{r}\right)=Q\left(\frac{y_{j}-\Delta / 2-f\left(y_{r}\right)}{\sqrt{N_{j}}}\right)-Q\left(\frac{y_{j}+\Delta / 2-f\left(y_{r}\right)}{\sqrt{N_{j}}}\right)$.

The remaining probability mass functions can be computed by marginalization.

The utility $U(\mathbf{f})$ is given by

$$
U(\mathbf{f})=I\left(X_{1} ; Y_{2} \mid X_{2}\right)+I\left(X_{2} ; Y_{1} \mid X_{1}\right) .
$$

The discretized version of the optimization problem (6) is given by

$$
\begin{array}{cl}
\text { maximize } & U(\mathbf{f}) \\
\text { subject to: } & \mathbf{f}^{T} \mathbf{Q f} \leq P_{r},
\end{array}
$$

where $\mathbf{Q} \succeq 0$ is a diagonal positive semidefinite matrix with the diagonal entries $Q_{i i}=\operatorname{Prob}\left(Y_{r}=y_{r i}\right) \geq 0$.

There are different ways to approach problem (8): The authors in [6] run a fixed-point iteration scheme that is based on the Lagrangian of problem (8). We take an alternative approach and find solutions via the projected gradient method [18, Sect. 2.3]. The update rule is

$$
\mathbf{f}^{k+1}=\mathcal{P}\left\{\mathbf{f}^{k}+\alpha_{k} \frac{\partial U\left(\mathbf{f}^{k}\right)}{\partial \mathbf{f}}\right\},
$$

where $\mathcal{P}\{$.$\} defines a projection onto the feasible set and \alpha_{k}$ denotes a suitably chosen diminishing stepsize.

The derivative with respect to a single element of the vector 
f, denoted $\frac{\partial U}{\partial f_{i}}$, is given by

$$
\begin{aligned}
\frac{\partial U}{\partial f_{i}} & \propto \sum_{x_{1}} \sum_{x_{2}} \sum_{y_{2}} p\left(y_{2}, y_{r i}, x_{1}, x_{2}\right) \frac{y_{2}-f_{i}}{N_{2}} \log \frac{p\left(y_{2} \mid x_{1}, x_{2}\right)}{p\left(y_{2} \mid x_{2}\right)} \\
& +\sum_{x_{1}} \sum_{x_{2}} \sum_{y_{1}} p\left(y_{1}, y_{r i}, x_{1}, x_{2}\right) \frac{y_{1}-f_{i}}{N_{1}} \log \frac{p\left(y_{1} \mid x_{1}, x_{2}\right)}{p\left(y_{1} \mid x_{1}\right)} .
\end{aligned}
$$

The update in Eq. (9) can be computed efficiently if the projection $\mathcal{P}\{$.$\} can be easily found. Fortunately, the feasible$ set $\mathcal{S}=\left\{\mathbf{f} \mid \mathbf{f}^{T} \mathbf{Q f} \leq P_{r}\right\}$ is convex and thus the projection can be expressed as finding the closest point $\mathbf{f}^{k+1} \in \mathcal{S}$ to $\overline{\mathbf{f}}^{k}=\mathbf{f}^{k}+\alpha_{k} \frac{\partial U}{\partial \mathbf{f}}$, corresponding to the following problem:

$$
\begin{aligned}
& \mathcal{P}\{.\} \text { : minimize }\left\|\mathbf{f}^{k+1}-\overline{\mathbf{f}}^{k}\right\| \\
& \text { subject to: } \quad \mathbf{f}^{k+1, T} \mathbf{Q} \mathbf{f}^{k+1} \leq P_{r} \text {. }
\end{aligned}
$$

We can solve this problem with a standard convex optimization solver such as CVX [19]. The update rule of the projected gradient ascent in Eq. (9) is stopped if $\left\|\mathbf{f}^{k+1}-\mathbf{f}^{k}\right\|$ is sufficiently small.

Depending on the initialization, we observe that the algorithm converges to different local optima resulting in different sum rates. It is therefore important to have a good starting point before launching the optimization procedure. In our simulations, we observed that initializations corresponding to the strategies summarized in the next section are a good choice. Additionally, the method can be used to check if a certain mapping is a local optimum.

\section{Strategies}

In this section, we summarize different memoryless strategies to be compared in Sec. VI.

\section{A. Amplify \& Forward (AF)}

The AF relay mapping is given by

$$
f_{A F}\left(y_{r}\right)=K y_{r}
$$

where the constant $K=\sqrt{\frac{P_{r}}{P_{1}+P_{2}+N_{r}}}$ ensures that the power constraint at the relay is satisfied. The received signal at source $T_{j}$ is given by $y_{j}=K\left(x_{j}+x_{i}+z_{r}\right)+z_{j}$.

After subtracting the own signal, this scheme can be interpreted as transmission over an AWGN channel with effective SNR of $\gamma_{A F, j}=\frac{K^{2} P_{j}}{K^{2} N_{r}+N_{i}}$ The achievable sum-rate is thus limited by $C\left(\gamma_{A F, 1}\right)+C\left(\gamma_{A F, 2}\right)$, where $C(\cdot)=\frac{1}{2} \log _{2}(1+\cdot)$ denotes the capacity for the one-dimensional AWGN channel with Gaussian input.

\section{B. Detect \& Forward (DF)}

A DF relay makes hard decisions on the received symbol. Assume both sources use the same modulation format with $M$ equally-spaced discrete constellation points. For the sum signal $V=X_{1}+X_{2}$, there are at most $M^{2}$ discrete points if each combination of the sum of input symbols is distinct. If both users have the same power $P_{1}=P_{2}$, the sum signal is identical for different individual input symbols, so there are only $2 M-1$ discrete points for $V$. The number of distinct superimposed transmit symbols $V \in \mathcal{V}$ is limited by $2 M-1 \leq|\mathcal{V}| \leq M^{2}$. One possible strategy is to assign one distinct transmit level $l_{j} \in \mathcal{L}$ to each different symbol $v_{j} \in \mathcal{V}$, i.e.

$$
f\left(y_{r}\right)=l_{j} \quad \text { if } \quad\left\|y_{r}-v_{j}\right\|<\left\|y_{r}-v_{j^{\prime}}\right\|, \forall v_{j} \neq v_{j^{\prime}} \in \mathcal{V} .
$$

We choose the transmit levels $l_{j}$ to be equally spaced and satisfy the power constraint at the relay. The number of different transmit levels $|\mathcal{L}|$ can be reduced if the side information available at the sources is taken into account. In the noiseless case, given either $X_{1}$ or $X_{2}$, there are only $M$ distinct possible sum symbols $V$. The number of transmit levels can thus be reduced to $|\mathcal{L}|=M$. As already pointed out in [5], one can define a function $g(v)$ that uniquely determines $x_{2}$ given $x_{1}$ and vice versa. Precisely, the function ensures that $H\left(X_{2} \mid g(V), X_{1}\right)=H\left(X_{1} \mid g(V), X_{2}\right)=0$.

The function $g(v)$ defines a network coding operation for the noiseless case. Finding such a function $g(v)$ corresponds to a graph coloring problem in a suitably defined graph, each color representing a certain transmit level. Due to space limitations, we refer to [5] for details, but provide a relevant example: For 4PAM and $P_{1}=P_{2}$, the set of possible sum symbols is given by $\mathcal{V}=\{-6 a,-4 a,-2 a, 0,2 a, 4 a, 6 a\}$ for some $a>0$ depending on the power. There is only one valid coloring with $M=4$ levels, corresponding to the partitioning $\mathcal{P}_{1}=\{-6 a, 2 a\}, \mathcal{P}_{2}=\{-4 a, 4 a\}, \mathcal{P}_{3}=\{-2 a, 6 a\}$, $\mathcal{P}_{4}=\{0\}$ with $\bigcup_{j=1}^{M} \mathcal{P}_{j}=\mathcal{V}$. One transmit level $l_{j}$ is assigned to each partition $\mathcal{P}_{j}$ :

$$
g(v)=l_{j}, \quad \text { if } v \in \mathcal{P}_{j}
$$

The DF relay mapping is thus defined as

$$
f_{D F}\left(y_{r}\right)=g(v), \quad \text { if }\left\|y_{r}-v\right\|<\left\|y_{r}-v^{\prime}\right\|, \forall v, v^{\prime} \in \mathcal{V} \text {. }
$$

Note that the assignment of transmit levels and partitions can be permuted, resulting in different sum rates. In our numerical results, we use the assignment with the best performance.

\section{Estimate \& Forward (EF)}

If the relay forwards an MMSE estimate of a function of the received signal, the strategy is called Estimate \& Forward [13]. For BPSK, we consider only the MMSE estimate of the absolute value of the symbol, like in [5], [15].

For $P_{1}=P_{2}=P$, the BPSK EF mapping is given by

$$
\begin{aligned}
f_{E F}\left(y_{r}\right) & =K\left(\mathbb{E}\left\{\mid X_{1}+X_{2} \| Y_{r}=y_{r}\right\}-c\right) \\
& =K\left(\frac{2 \sqrt{P} \cosh \left(\frac{2 \sqrt{P} y_{r}}{N_{r}}\right)}{e^{2 P / N_{r}}+\cosh \left(\frac{2 \sqrt{P} y_{r}}{N_{r}}\right)}-c\right) .
\end{aligned}
$$

$K$ again denotes a constant to take into account the power constraint. The constant shift $c$ is chosen to minimize the relay signal power. For PAM, we consider the EF mapping like in [5], which is defined as the MMSE estimate of a function of the input signal 


$$
f_{E F}\left(y_{r}\right)=K\left(\mathbb{E}\left\{g\left(X_{1}+X_{2}\right) \mid Y_{r}=y_{r}\right\}-c\right)
$$

with $g(\cdot)$ as defined in Eq. (14).

An example of a DF mapping and corresponding EF mapping for 4PAM is shown in Fig. 1.

\section{UPPER BOUNDS}

Based on the cut-set bound [20], the following bounds can be obtained:

$$
\begin{aligned}
& R_{1}<\min \left\{I\left(X_{1} ; Y_{r} \mid X_{2}\right), I\left(X_{r} ; Y_{2}\right)\right\} \\
& R_{2}<\min \left\{I\left(X_{2} ; Y_{r} \mid X_{1}\right), I\left(X_{r} ; Y_{1}\right)\right\}
\end{aligned}
$$

The channels corresponding to $I\left(X_{1} ; Y_{r} \mid X_{2}\right)$ and $I\left(X_{r} ; Y_{2}\right)$ are AWGN channels for which Gaussian inputs give a capacity upper bound. The achievable rates are thus limited by

$$
\begin{aligned}
& R_{1}<\min \left\{C\left(P_{1} / N_{r}\right), C\left(P_{r} / N_{2}\right)\right\} \\
& R_{2}<\min \left\{C\left(P_{2} / N_{r}\right), C\left(P_{r} / N_{1}\right)\right\}
\end{aligned}
$$

We include the upper bounds in the plots in Fig. 2c and Fig. 2d.

\section{Numerical Results}

We investigate the achievable sum rates of the relay mappings given in Sec. IV. Moreover, we check whether these mappings can be further improved by using them as initial values for the optimization routine presented in Sec. III.

We investigate two different scenarios:

- In Scenario 1, uplink and downlink SNR are simultaneously scaled, i.e. $P_{1}=P_{2}=P_{r}$, $N_{1}=N_{2}=N_{r}$. The SNR $P_{1} / N_{1}$ ranges from 0 to $20 \mathrm{~dB}$.

- In Scenario 2, the downlink SNR is fixed at $P_{r} / N_{1}=$ $P_{r} / N_{2}=12 \mathrm{~dB}$. The symmetric uplink SNR $P_{1} / N_{r}=$ $P_{2} / N_{r}$ ranges from 0 to $20 \mathrm{~dB}$.

A uniform quantizer with 64 intervals is used at the relay.

For Scenario 1 and BPSK signaling, results are shown in Fig. 2a. We plot the achievable sum rate for each strategy described in Sec. IV. Additionally, we plot the sum rate achieved by the locally optimal relay mapping that was found by taking $\mathrm{AF}, \mathrm{DF}$ and $\mathrm{EF}$ as initialization. We observe that $\mathrm{EF}$ dominates $\mathrm{DF}$ and $\mathrm{AF}$ over the whole SNR range. Furthermore, we observe that the local optimum starting from $\mathrm{AF}$ as initial value is still worse than DF and EF. The local optimum found when starting from DF is essentially equal to $\mathrm{EF}$. With EF as initialization, no significant improvement can be achieved. We could not find a mapping with higher sum rate than EF as defined in Eq. (16) for BPSK in this scenario.

Considering 4PAM in Fig. 2c, we see that surprisingly AF is superior to both DF and EF for low SNR. However, we also note that the local optimum found with EF as initialization essentially achieves the same sum rate. The EF optimum is close to the best observed performance over the whole SNR range. An example of initial $\mathrm{EF}$ and $\mathrm{DF}$ mappings and the corresponding local optimum is shown in Fig. 1. In this case, essentially the same optimum is achieved. We also note that BPSK results in better rates than 4PAM and Gaussian signaling for low SNR. This could be adapted by optimizing the input distribution of 4PAM.

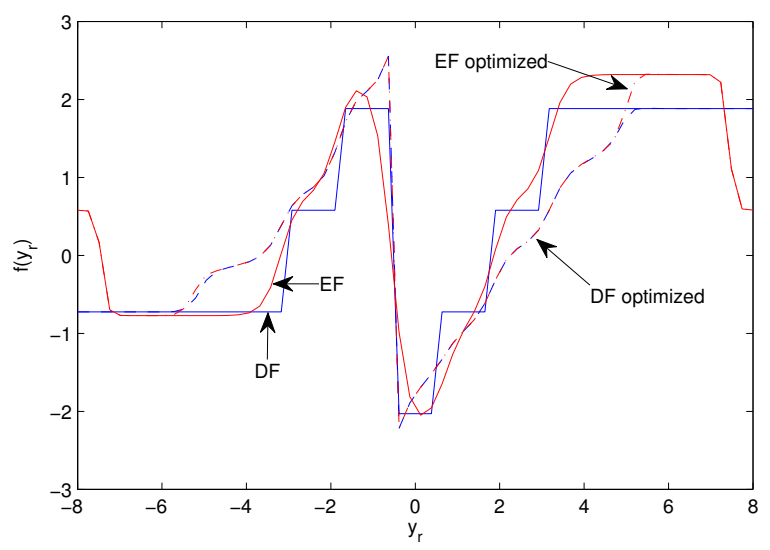

Fig. 1: Relay Mappings for DF and EF for 4-PAM in Scenario 1 and a SNR of $9 \mathrm{~dB}$.

For Scenario 2, BPSK results are shown in Fig. 2b. Unlike Scenario 1, EF is not optimal here as AF gives better sum rates for low SNR. For higher SNR, EF becomes the best observed mapping. Unfortunately, no local optimum is optimal over the whole SNR range. Similarly for 4PAM in Fig. 2d: $\mathrm{AF}$ is superior to $\mathrm{EF}$ for low SNR. At approximately $12 \mathrm{~dB}$, as the downlink starts having smaller SNR than the uplink, $\mathrm{EF}$ dominates AF again. We observe that the local optimum found with EF as initial value performs well for low and high SNR. Interestingly, Gaussian signaling combined with $\mathrm{AF}$ is suboptimal and significantly worse than EF for 4PAM.

\section{CONCLUSIONS AND FUtURE WORK}

We investigated the behaviour of memoryless relaying strategies for the two-way relay channel in terms of achievable sum rate. We compared state-of-the-art memoryless relay mappings and proposed numerical methods to optimize their performance. Our results suggest that using EF as an initialization for the optimization is a good choice in most cases.

In general, finding an optimal mapping analytically would be highly desirable but seems intractable. Likewise, analytically determining SNR ranges for which a certain memoryless strategy is superior to others would be highly useful and is the focus of our future work.

\section{ACKNOWLEDGEMENT}

The work was supported by the German Federal Ministry of Education and Research in the framework of the Alexander von Humboldt-Professorship and by the grant DLR@Uni of the Helmholtz Allianz. The authors thank Prof. Gerhard Kramer for the helpful discussions.

\section{REFERENCES}

[1] B. Rankov and A. Wittneben, "Achievable rate regions for the two-way relay channel," in IEEE Int. Symp. on Inf. Theory (ISIT), July 2006, pp. 1668-1672. 


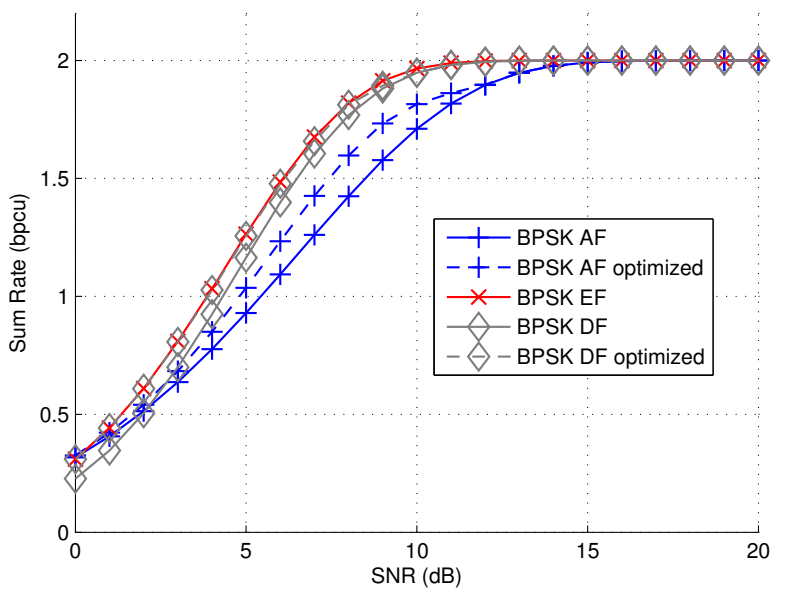

(a) BPSK curves for Scenario 1

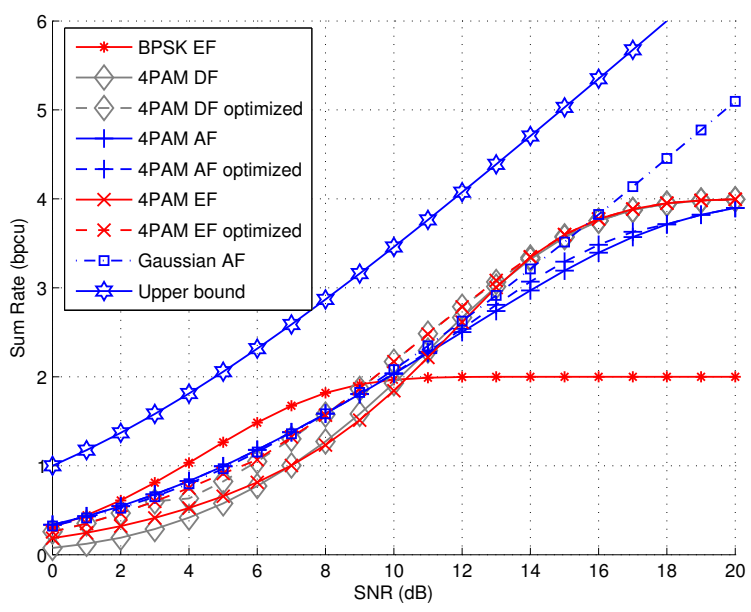

(c) Overview for Scenario 1

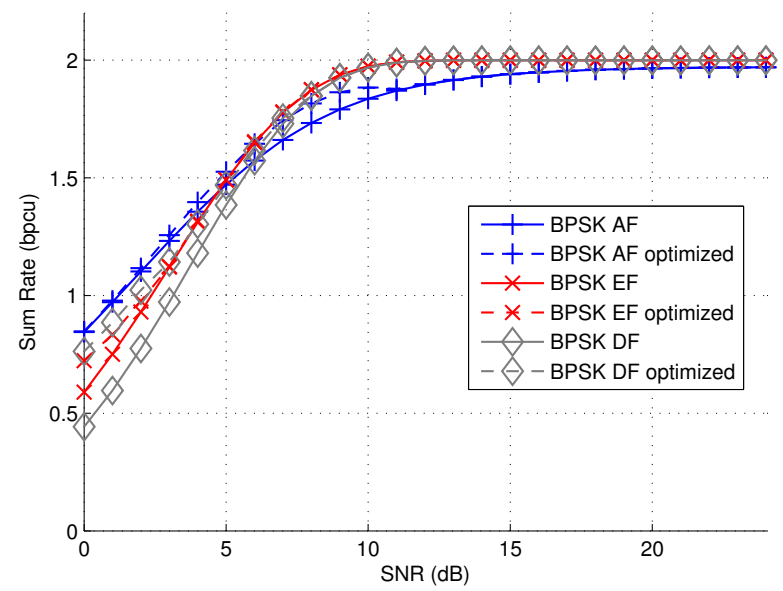

(b) BPSK curves for Scenario 2

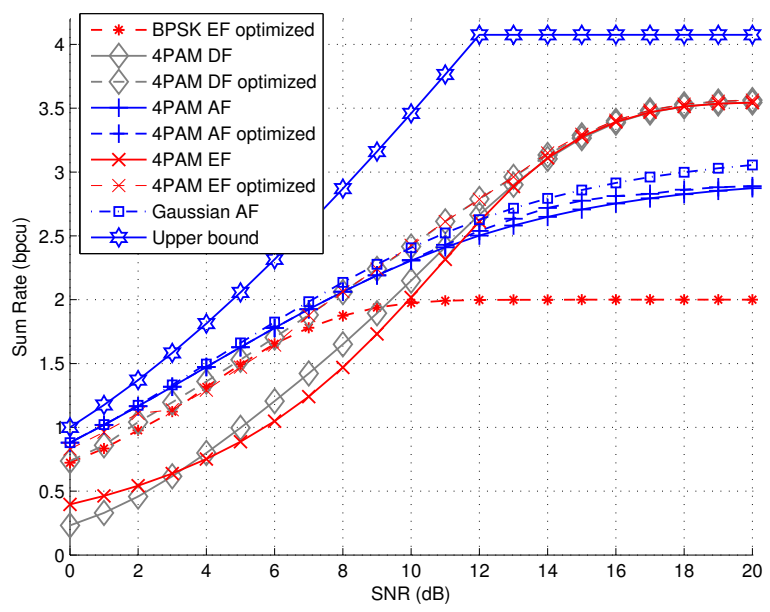

(d) Overview for Scenario 2

Fig. 2: Overview of numerical results. All sum rates are expressed in bits per channel use.

[2] B. Nazer and M. Gastpar, "Computation over multiple-access channels," IEEE Transactions on Information Theory, vol. 53, no. 10, pp. 3498 -3516 , oct. 2007.

[3] K. Narayanan, M. Wilson, and A. Sprintson, "Joint physical layer coding and network coding for bi-directional relaying," in 45th Annual Allerton Conference on Communication, Control, and Computing, 2007.

[4] B. Nazer and M. Gastpar, "Reliable physical layer network coding," Proceedings of the IEEE, vol. 99, no. 3, pp. 438-460, 2011.

[5] T. Cui, T. Ho, and J. Kliewer, "Memoryless relay strategies for two-way relay channels," IEEE Transactions on Communications, vol. 57, no. 10, pp. 3132-3143, Oct. 2009.

[6] M. Khormuji and M. Skoglund, "Noisy analog network coding for the two-way relay channel," in IEEE Int. Symp. on Inf. Theory (ISIT), 2011, pp. 2065-2069.

[7] S. Zhang, S. Liew, and P. Lam, "Hot topic: physical-layer network coding," in Proceedings of the 12th annual international conference on Mobile computing and networking. ACM, 2006, pp. 358-365.

[8] S. Katti, S. Gollakota, and D. Katabi, "Embracing wireless interference: Analog network coding," in ACM SIGCOMM Computer Communication Review, vol. 37, no. 4. ACM, 2007, pp. 397-408.

[9] P. Popovski and H. Yomo, "The anti-packets can increase the achievable throughput of a wireless multi-hop network," in IEEE Int. Conference on Communications (ICC), vol. 9, june 2006, pp. 3885 -3890.

[10] M. Khormuji and M. Skoglund, "On instantaneous relaying," IEEE Trans. on Information Theory, vol. 56, no. 7, pp. 3378-3394, 2010.
[11] B. Rankov and A. Wittneben, "Spectral efficient protocols for half-duplex fading relay channels," IEEE Journal on Selected Areas in Communications, vol. 25, no. 2, pp. 379-389, 2007.

[12] I. Abou-Faycal and M. Medard, "Optimal uncoded regeneration for binary antipodal signaling," in IEEE International Conference on Communications (ICC), vol. 2, June 2004, pp. 742-746.

[13] K. Gomadam and S. Jafar, "On the capacity of memoryless relay networks," in IEEE International Conference on Communications (ICC), vol. 4, June 2006, pp. 1580-1585.

[14] P. Weitkemper and G. Dietl, "Maximum likelihood receiver for MMSE relaying," in IEEE International Conference on Communications (ICC), June 2011, pp. 1-5.

[15] O. Iscan, M. Heindlmaier, and C. Hausl, "Network coded two-way relaying with reduced relay complexity," in 8th International Symposium on Wireless Communication Systems (ISWCS), 2011, pp. 497-501.

[16] D. Gunduz, E. Tuncel, and J. Nayak, "Rate regions for the separated two-way relay channel," in 46th Annual Allerton Conference on Communication, Control, and Computing, Sept. 2008, pp. 1333-1340.

[17] C. Shannon, "Two-way communication channels," in Proc. 4th Berkeley Symp. Math. Stat. Prob, vol. 1, 1961, pp. 611-644.

[18] D. Bertsekas, Nonlinear programming. Athena Scientific, 1999.

[19] M. Grant and S. Boyd, "CVX: Matlab software for disciplined convex programming, version 1.22," http://cvxr.com/, Feb. 2012.

[20] T. Cover and J. Thomas, Elements of Information. John Wiley \& Sons, New Jersey, 2006. 